\title{
Bistatic Radar Signature of Buried Landmines
}

\author{
F. Lombardi*, H.D. Griffiths*, A. Balleri ${ }^{\dagger}$ \\ * Department of Electronic \& Electrical Engineering, University College London, London WC1E 6BT, United Kingdom, \\ f.lombardi@ucl.ac.uk \\ ${ }^{+}$Centre for Electronic Warfare, Information and Cyber, Cranfield University, Defence Academy of the UK, Shrivenham SN6 \\ 8LA, United Kingdom
}

Keywords: Ground Penetrating Radar, Landmine detection, Signature characterisation, Bistatic GPR.

\begin{abstract}
With the proliferation of low-intensity conflict, landmines have proven to be one of the weapons of choice for both government and guerrilla forces around the world. Recent improvements to mine technology pose increasingly significant problems for demining operations, requiring the constant upgrading of countermine technologies. Ground Penetrating Radar (GPR) is one of the most exhaustively researched topics in the detection of buried mines as it can be used to detect non-metallic and plastic mines. However, identification and recognition are still unsolved problems, due to the scattering similarity between mines and clutter objects. This study provides an experimental evaluation of the improvements that a bistatic approach could yield and what can be gained from investigating the angular dependencies of the landmine radar signature.
\end{abstract}

\section{Introduction}

Today the plague of landmines has enveloped the world's conflict zones. With the proliferation of low-intensity conflict, landmines have proven to be one of the weapons of choice in civil wars and wars of insurgency for both government and guerrilla forces around the world [1], [2]. It is not possible to provide a global estimate of the total area contaminated by landmines due to a lack of data, however, 11 states have been identified as current producers of antipersonnel landmines (APL), with many more reserving the right to do so, and more than 60 countries and areas are contaminated by mines [3]. Landmines represent one of the greatest curses of modern time, killing and maiming innocent people every day. Reported casualties describe that the majority of the victims are civilians, with almost a half represented by children [4], [5].

Amongst other geophysical investigation methods, Ground Penetrating Radar (GPR) appears to be a promising candidate, as it allows non-invasive and cost-effective surveys to be undertaken, and has the advantage of a high resolution imaging capability [6][7][8]. GPR operates by transmitting an electromagnetic signal into the subsurface and detecting a target echo at a receiver antenna, that is reflected due to the dielectric discontinuity between the target and the propagation medium. GPR has a wide range of applications in archaeology [9], [10], engineering [11], [12][13] and geological applications [14], [15].

One of the problems with GPR for landmine detection is that dielectric discontinuities occur at places other than the mine, such as roots, rocks and hollows, as well as other battlefield debris. These reflections can hide the existence of a mine by cluttering the return signal and provide false alarms [16].

Typical GPR surveys are collected in common offset mode, where one transmitting and one receiving antenna move together along the surface keeping a constant offset [17], [18]. Generally, such configuration is also reported as monostatic or quasi-monostatic because the two antennas are almost colocated. Although the majority of experimental trials have been performed following this approach, a bistatic geometry, in which the transmitter and the receiver are independently managed, may offer several key benefits, especially for lowobservable targets or low SNR scenarios [19], [20]. For example, targets designed to minimise backscatter might be easily detected by a bistatic configuration. Objects with irregular or rough shape could reflect the incident wave in a particular direction far from the monostatic receiver, thus multiple looks at a target from a variety of antenna spacing could make it easier to distinguish target of interest from clutter features [21], [22][23]. Finally, changing the transmitter and receiver distance can better highlight targets with composite structure and internal assemblies.

Many of the newer landmines are deliberately constructed to defeat metal detectors, containing only the barest minimum of metal and using materials such as plastic, wood or glass. As a consequence, their detection and discrimination from objects causing false alarms can be improved by increasing the information extracted from their radar signature [24], [25].

In opposition to metallic targets, a variation of the separation between antennas will illuminate a progressively different internal section of the target, generating a signature clearly affected by the characteristics of that particular area.

Employing a number of representative inert landmines buried in a sharp sand environment, the paper presents the results of a preliminary characterisation of the bistatic signature of buried landmine to demonstrate that such approach can effectively enhance the knowledge of the features of the detected target and potentially highlight the presence of internal structures.

\section{Target and acquisition description}

A set of bistatic signatures from three different inert landmines has been acquired in a test sand pit located at the 
Defence Academy of the United Kingdom in Shrivenham (Figure 1a). The sharp sandy material of the pit is characterised by a very low clay content and a gritty texture for a better drainage and to avoid trench effects during digging operations (Figure 1b).

(a)

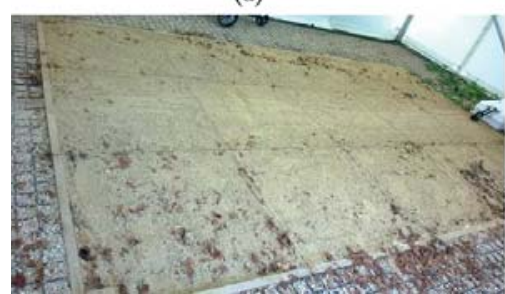

Figure 1: Experimental environment. (a) Test pit. (b) Host material.

The GPR equipment employed for the measurements consisted of an IDS Aladdin radar and an IDS THRHF radar, both provided by IDS Georadar srl. The two impulsed devices carry dipole antennas with a central frequency and a bandwidth of $2 \mathrm{GHz}$ and $3 \mathrm{GHz}$, respectively. A soft pad, the PSG (Pad System for Georadar, U.S. Patent no. US 7,199,748 B2 of Politecnico di Milano, Italy, [26]), was placed between the radar equipment and the soil to ensure a better coupling and controlled antenna orientation from trace to trace. The measurement set-up is pictured in Figure 2. (a)

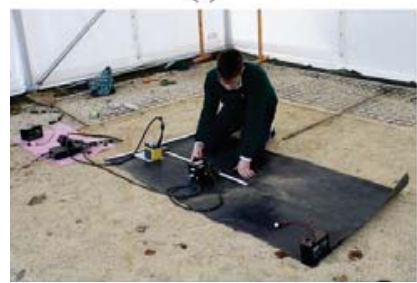

(b)

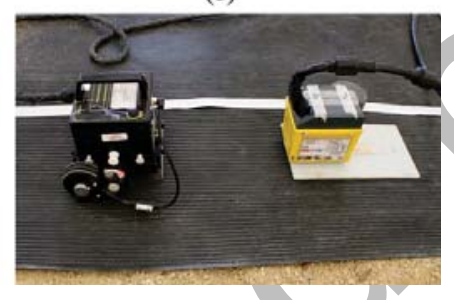

Figure 2: Measurement details. (a) Data acquisition. (b) Employed GPR devices.

Two assumptions are accepted in almost every landmine detection operations: the need of very high frequencies and the necessity of keeping the antennas away from the terrain surface [27], [28]. The first assumption is motivated by the goal of being able to classify the targets according to their accurate geometric reconstruction. The second assumption is a standard accepted approach for safety reasons. However, a lot of emitted energy is reflected back because the antenna is suspended above the surface and the coupling process efficiency is lower [29], [30][31]. Figure 3 provides an example of the energy and resolution losses experienced by elevating the antenna of few centimetres above the surface.

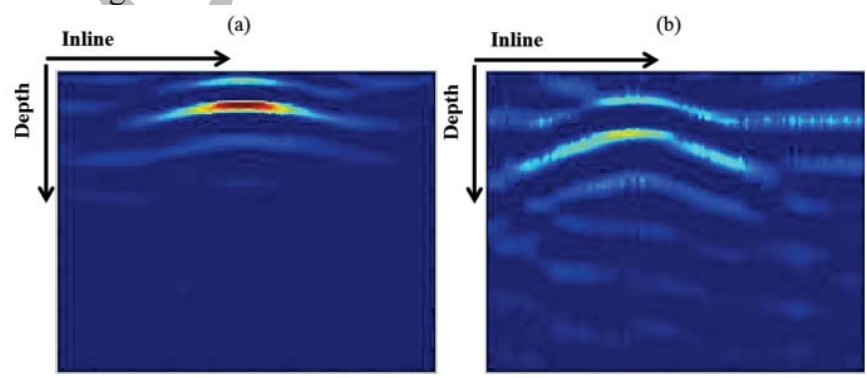

Figure 3: Comparison of raw data from (a) ground coupled and (b) stand-off radar ( $4 \mathrm{~cm}$ above the surface).
The contact between the antenna and the terrain is essential to maximise the penetration of the radar signal and to ensure proper performance. In addition, rough air-soil interface scatters GPR waves randomly, making the received data difficult to analyse, while when the antennas are in contact with the ground, the subsurface waveform is nearly unaffected by the roughness of the soil and therefore is easier to predict. As a consequence, data processing too is positively affected by a ground coupling survey [32], [33].

The ensemble of bistatic signatures has been collected by progressively shifting both the transmitter and the receiver away from the target location, known as Common Mid Point (CMP) acquisition. The CMP sounding is completed by progressively increasing the transmitter/receiver separation (offset) of the antennas in steps relative to the selected midpoint location along the original profile. The process is sketched in Figure 4. An accurate positioning has been achieved employing an odometric wheel (visible in Figure $2 b)$.

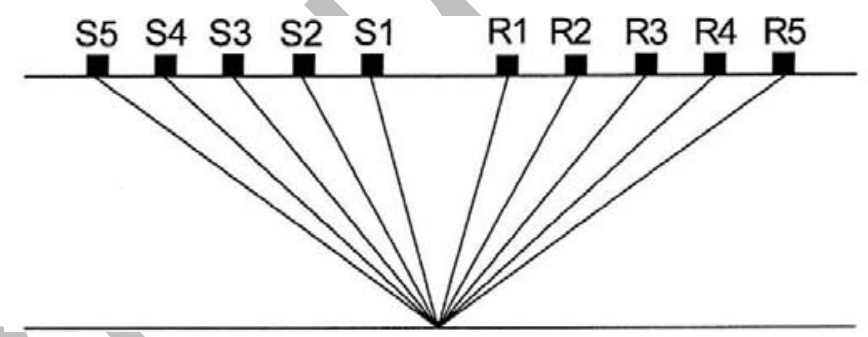

Figure 4: CMP acquisition scheme. $S_{i}$ represents the source location, while $R_{i}$ stands for the receiver position.

The higher frequency equipment acted as the receiver module to take advantage of the finer sensitivity of its components. The acquisition details are provided in Table 1.

\begin{tabular}{|l|l|}
\hline Parameter & Value \\
\hline Separation range & $6-33 \mathrm{~cm}$ \\
Offset increment & $1 \mathrm{~cm}$ \\
Time window & $30 \mathrm{~ns}$ \\
Time sampling & $0.0587 \mathrm{~ns}$ \\
\hline \multicolumn{2}{|c|}{ Table 1: Acquisition details. }
\end{tabular}

No processing steps have been computed on the data to preserve their original features and to avoid possible artefacts [34].

It is essential that properly constructed inert landmines are used for research and development, otherwise the results could be significantly affected or misleading. For the purpose of this research, a number of representative landmine models, provided by the Defence Academy of the United Kingdom, were used and their bistatic signature was acquired.

These were complete with all their external and internal components and were filled with a high explosive simulant commonly used to train the UK Ammunition Technical Officers; the substance has the same electrical and chemical properties of commonly employed explosive materials.

In particular, the Italians VS-50 and SB-33, and a Soviet PFM-1 devices were investigated. Targets are pictured in 
Figure 5, and their geometrical features are described in Table $2[35]$.

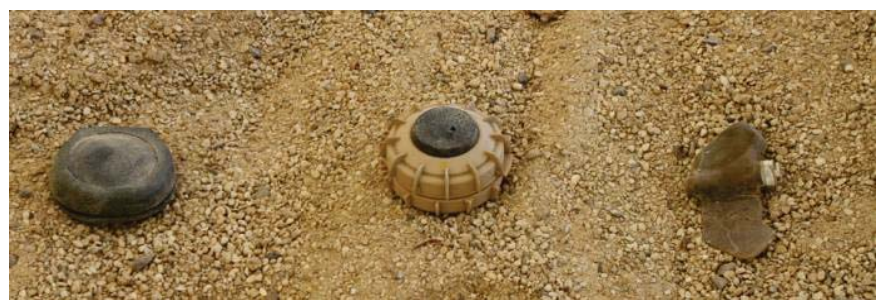

Figure 5: Investigated targets. From left to right: SB-33, VS50 and PFM-1 model.

\begin{tabular}{|l|c|}
\hline Model & $\begin{array}{c}\text { Length/Width/Height } \\
{[\mathrm{mm}]}\end{array}$ \\
\hline SB-33 & $85 / 85 / 30$ \\
VS-50 & $90 / 90 / 45$ \\
PFM-1 & $120 / 20 / 61$ \\
\hline
\end{tabular}

Table 2: Targets descriptions.

Targets were buried at approximately $10 \mathrm{~cm}$, with their activator plate pointing toward the surface, as shown in Figure 5.

Special reference needs to be made to the scatterable PFM-1 landmine, which is in reality filled with a liquid explosive and not with a solid mixture. However, this limitation is negligible for the scope of this study.

Moreover, as the main purpose was to evaluate the efficacy of this approach for detecting internal scattering mechanisms, the choice of these particular targets answers the need of having a group of devices with different design and structure (Figure 6).
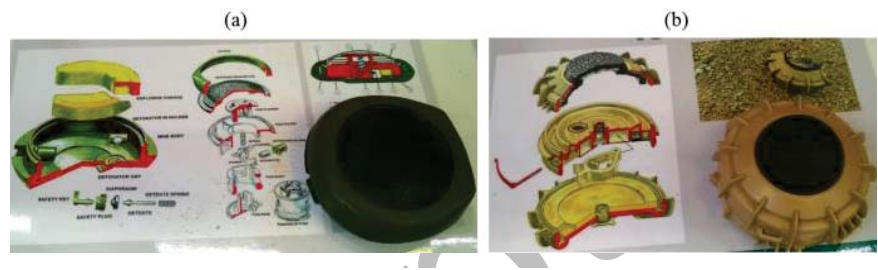

Figure 6: Details of the target design. (a) SB-33 and (b) VS50 device. Courtesy of Cranfield University.

\section{Results}

Bistatic signatures of landmines are presented in the commonly employed time/range versus offset display. The time axis represents the two way traveltime between the transmitter and the target. The range indication is the resulting inversion. The word offset, instead, is referred to the distance between the transmitting antenna and the receiving one. Figure 7 provides an example of a bistatic radar image and what can be observed from a visual analysis.

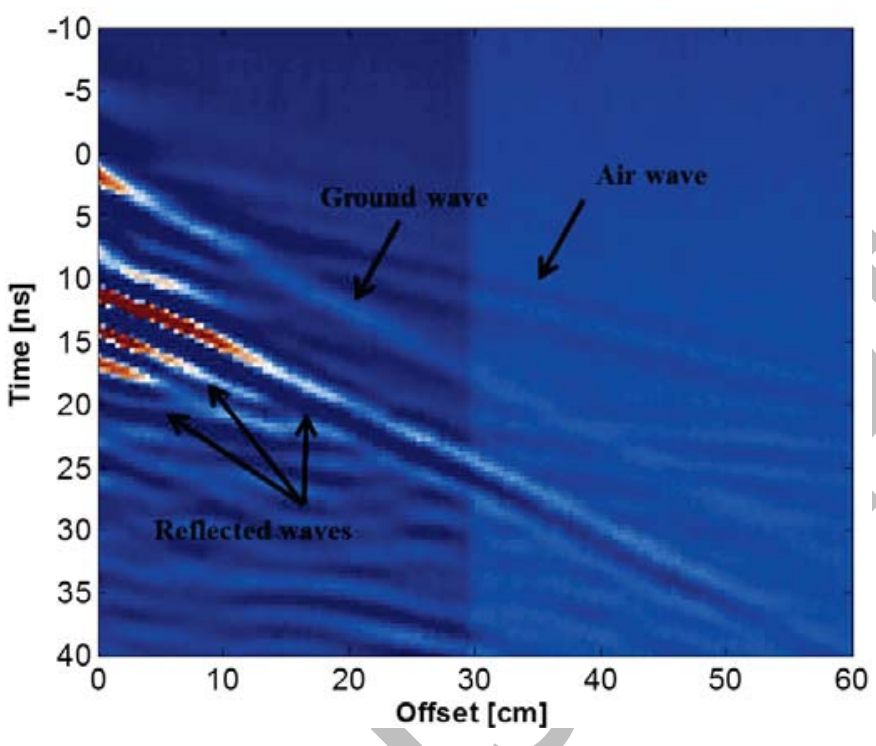

Figure 7: Example of bistatic radar image.

Generally, bistatic images include three well determined events: (1) the air wave, which is the wave that travels directly from the transmitter towards the receiver, (2) the ground wave, which is the wave propagating over the surface, and (3) the reflections generated by the target scattering. The gradient of the detected events is inversely proportional to the electrical properties of the material in which the wave is propagating; therefore, for a target buried in a homogeneous medium, the latter two events have the same slope.

The bistatic angle can be computed accordingly from the trigonometric relationship between the transmitter/receiver offset and the target depth, as shown in Figure 8.

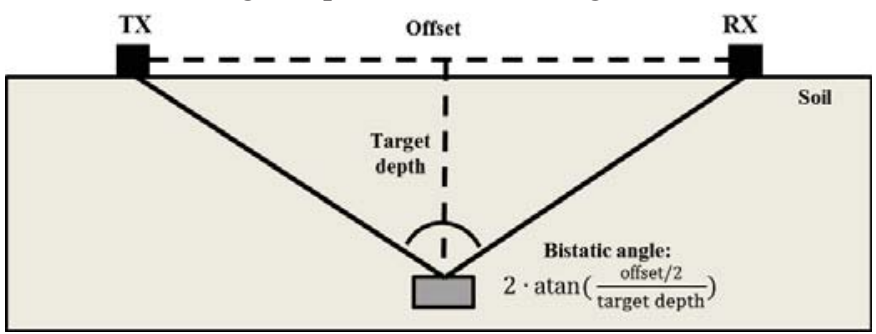

Figure 8: Trigonometric equivalences for bistatic angle calculation.

Results of the PFM-1 landmine are displayed in Figure 9. A single reflection is visible (marked A), with a spatial extension directly linked to the physical dimension of the target, and no further events are detectable. Given the nature of the target (a solid dielectric component), this was expected. 


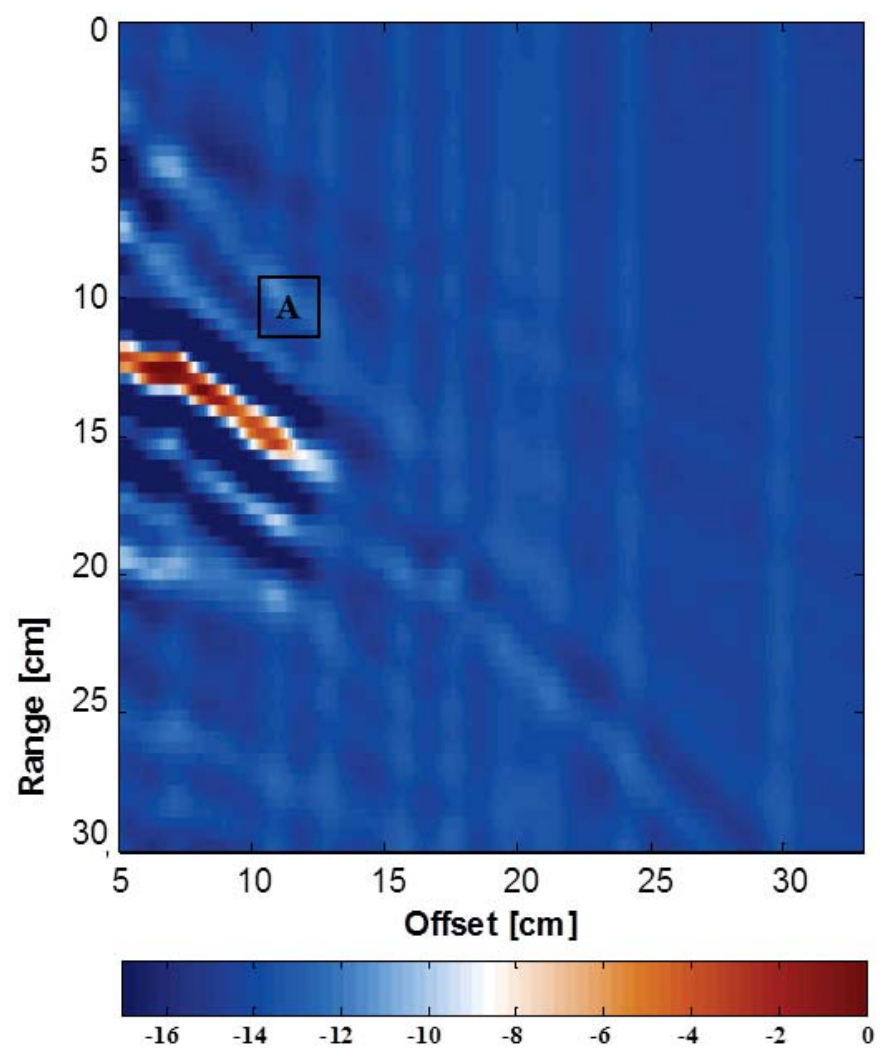

Figure 9: Bistatic signature of the PFM-1 landmine.

The situation changes when the illuminated target includes internal assemblies, which is the case for the following devices. Because the antenna separation controls the vertical position of the reflection plane, as described before, the presence of a structure beneath the top layer of the mine will generate an additional scattering feature which hopefully would be stronger under particular incident angles.

The bistatic signatures of the VS-50, shown in Figure 10, support these hypotheses. In this case, three events are detectable, and these have almost the same spatial extension. While the upper and lower reflections are due to the top and the bottom of the landmine (marked $\mathrm{A}$ and $\mathrm{C}$, respectively), the middle one is generated from an internal scattering point. Its constant trend over the separation range assumes an internal layer covering the whole landmine extension. Considering the design of the target, Figure $6 \mathrm{~b}$, the detected multiple reflections is due to the presence, below the activator plate, of a sunburst of air gaps, which allow the detonation to take place.
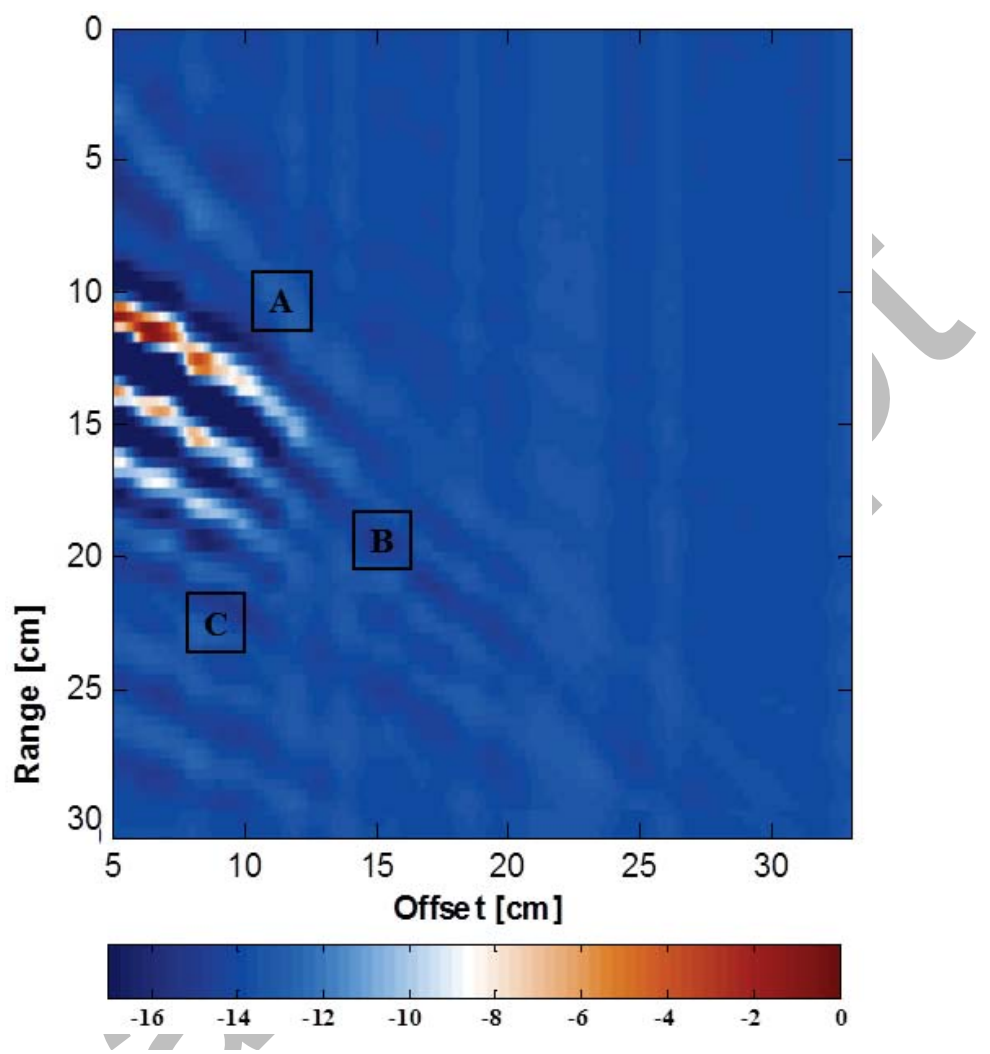

Figure 10: Bistatic signature of the VS-50 landmine.

In opposition to the previously described targets, the SB-33 landmine presents a highly heterogeneous internal design, as can be hinted from Figure 6a. Their ranges versus offset results are provided in Figure 11.

In a similar manner to the VS-50, more than one reflection is evident, therefore a preliminary indication of a target with a composite structure can be obtained. However, the middle reflection (marked B) is spatially longer than the top (marked A) and bottom one (marked D), demonstrating that the scattering event is not homogeneous over the target space. This reflection is due to the void located aside the detonator (identifiable with reflection $\mathrm{C}$ ) which is located in a particular section of the target. In this case, the advantage of a bistatic approach is clearly visible, as this reflection is stronger under a particular angular range, differently from the other reflections. Finally, as the extension of the upper and lower reflections is a marker of the target physical dimension, in this case a smaller object is identified, in agreement with the characteristics detailed in Table 2. 


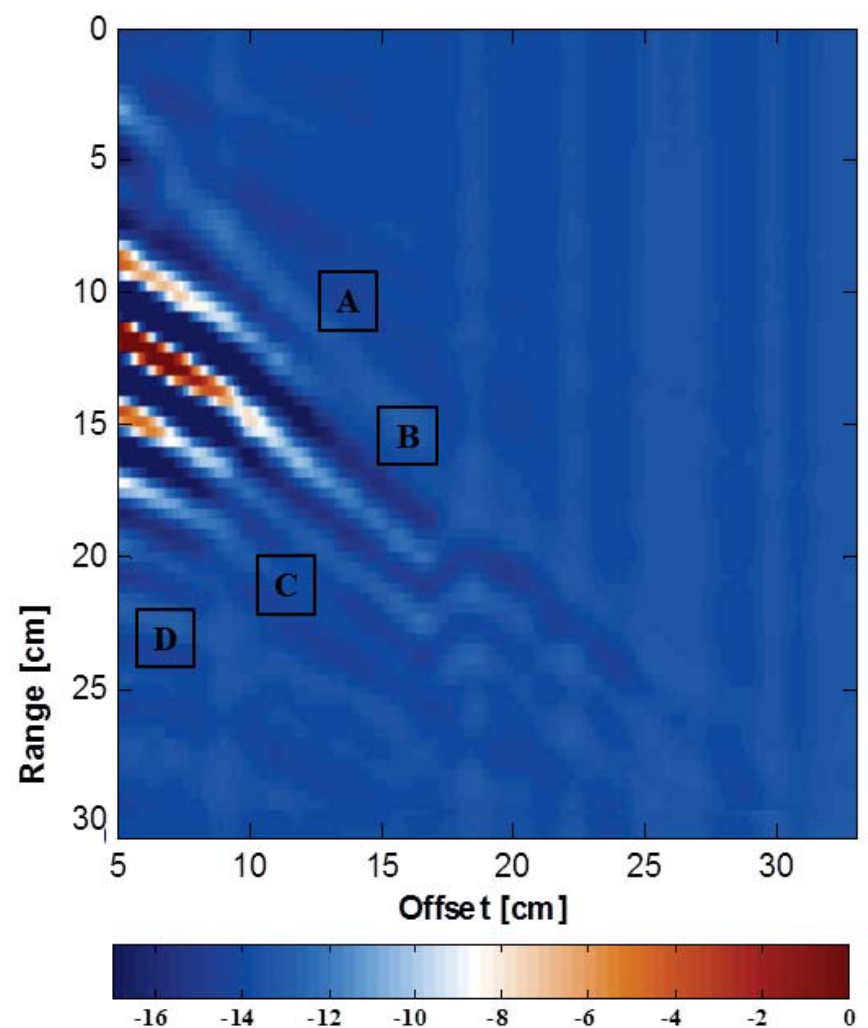

Figure 11: Bistatic signature of the SB-33 landmine.

For the last two devices, the VS-50 and the SB-33 landmine, an interpretative sketch is provided in Figure 12 to aid the recognition of the described events. (a)

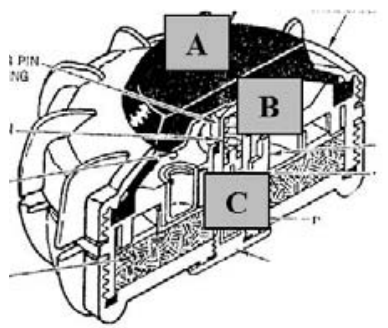

(b)

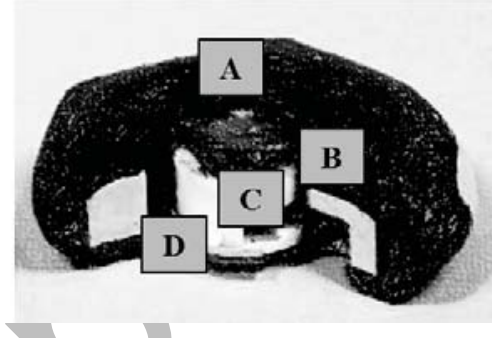

Figure 12: Interpretative diagrams (a) VS-50 and (b) SB-33 $[35]$.

\section{Conclusions}

The presented research has investigated the capabilities and advantages of a bistatic approach for landmine recognition. In particular, three different representative landmines have been investigated, each of them with a different external and internal design. The dielectric nature of the targets suggests that a change in the distance between the transmitter and the receiver will focus on a different internal section of the objects. Hence, bistatic GPR could possibly detect and identify a potential hazardous target based on its internal design.

As the capability of properly detect these internal reflections relies on a high resolution data, a high frequency and ground coupled GPR platform was employed for the experimental campaign. The direct contact with the surface increases the energy coupling process efficiency, limiting the loss of the higher frequencies due to the air-ground interface scattering, therefore ensuring a high quality collected data.

The outcomes have demonstrated that acquiring the signature changing the transmitter and receiver separation could yield additional information on the eventual presence of internal components, feature which is unlikely to be present in commonly encountered clutter objects. Hence, the possibility of detecting this feature, which can be considered as a discriminant characteristic, could significantly improve the performance of GPR and enhance its deployment as a landmine detection sensor.

Considering the three devices under investigation, there is a high correlation between the design of the internal assemblies and the spatial extension of the related radar reflection. In particular, when the target does not present any internal assemblies, as for the PFM-1, no additional reflections are detectable from a bistatic analysis. When the object is characterised by an elaborate design, instead, the contributions of these components is clearly visible in their radar signature.

Even if the PFM-1 landmine cannot be considered as a composite object, and closer to a clutter target, these results should be compared to the equivalent signatures of clutter targets, to further demonstrate the efficacy of this acquisition approach.

\section{Acknowledgements}

The authors thank the Find A Better Way charity for their support of this research under the DETERMINE programme (grant number 2015/001D). We also thank the Defence Academy Ammunition Hall for providing the real landmines used for the experiments.

\section{References}

[1]. 'International Campaign to Ban Landmines'. Available at http://www.icbl.org/

$$
\begin{array}{lcc}
\text { 'BBC Online, Available } \\
\text { http://www.bbc.com/news/uk-39487309 }
\end{array}
$$

[3]. 'Landmine and Cluster Munition Monitor'. Available at www.themonitor.org/

[4]. 'UN News Centre'. Available at http://www.un.org/apps/news/story.asp?NewsID=54 736

[5]. 'BBC News', Available at http://www.bbc.com/news/world-38070207

[6]. Bruschini, C., Bertrand, G.: "A survey of research on sensor technology for landmine detection", Journal of Conventional Weapons Destruction, 2(1), pp. 125, (2016).

[7]. D. Daniels, Ground Penetrating Radar, IEE ed., 2004.

[8]. D.W. Paglieroni, D.H. Chambers, J.E. Mast, S.W. Bond, N.R. Beer. "Imaging Modes for Ground Penetrating Radar and Their Relation to Detection Performance", IEEE Journal on Selected Topics in 
Applied Earth Observation and Remote Sensing, 8(3), pp. 1132-1144, (2015).

[9]. M. Lualdi, F. Lombardi, "Effects of antenna orientation on 3-D ground penetrating radar surveys: an archaeological perspective", Geophysical Journal International, 196(2), pp. 818-827, (2014).

[10]. L.B. Conyers, K.L. Kvamme (Ed.), "Groundpenetrating radar for archaeology". Altamira Press (2013).

[11]. M. Lualdi, F. Lombardi, "Utilities detection through the sum of orthogonal polarization in $3 \mathrm{D}$ georadar surveys, "Near Surface Geophysics, 13(1), pp. 7381, (2015).

[12]. L.B. Ciampoli, A. Benedetto, L. Pajewski, A.M. Alani, F. Tosti. "A comparison between different central frequencies of investigation in buried utility detection through GPR: A study case", 16th International Conference on GPR, pp. 1-4, (2016).

[13]. M. Lualdi, F. Lombardi, "Combining orthogonal polarisation for elongated target detection with GPR", Journal of Geophysics and Engineering, 11(5), 055006, (2014).

[14]. J.L. Davis, A.P. Annan "Ground Penetrating Radar for high resolution mapping of soil and rock stratigraphy", Geophysical Prospecting, 37(5), pp. 531-551, (1989).

[15]. C. Dorn, N. Linde, J. Doetsch, T. Le Borgne, O. Bour, "Fracture imaging within a granitic rock aquifer using multiple-offset single-hole and crosshole GPR reflection data", Journal of Applied Geophysics, 78, pp. 123-132, (2012).

[16]. D. J. Daniels, "A review of GPR for landmine detection", Sensing and Imaging: An international journal, 7(3), pp. 90-123, (2006).

[17]. E. Forte, M. Pipan,"Review of multi-offset GPR applications: Data acquisition, processing and analysis",Signal Processing,132,pp.210-220, (2016).

[18]. Tarantola, A. "Inverse problem theory and methods for model parameter estimation", SIAM ed., 2005.

[19]. G. Hamann, J. Tronicke, C.M. Steelman, A.L. Endres, "Spectral velocity analysis for the determination of ground-wave velocities and their uncertainties in multi-offset GPR data". Near Surface Geophysics, 11(2), pp. 167-176, (2013).

[20]. Sato, M., "Bistatic GPR system for landmine detection using optical electric field", IEEE Antennas and Propagation Society International Symposium, pp. 207-210, (2003).

[21]. T. Counts, A.C. Gurbuz, W.R. Scott, J.H. McClellan, K.Kim, "Multistatic ground-penetrating radar experiments". IEEE Transactions on Geoscience and Remote Sensing, 45(8), pp. 2544-2553, (2007).

[22]. Stiles, J. M., Parra-Bocaranda, P., Apte, A., "Detection of object symmetry using bistatic and polarimetric GPR observations", SPIE - Detection and Remediation Technologies for Mines and Minelike Targets, pp. 992-1002, (1999).

[23]. Jin, T., Lou, J., Zhou, Z., "Extraction of landmine features using a forward-looking ground-penetrating radar with MIMO array". IEEE Transactions on Geoscience and Remote Sensing, 50(10), pp. 41354144, (2012).

[24]. Lombardi, F., Griffiths, H. D., Wright, L., Balleri, A. "Dependence of landmine radar signature on aspect angle". IET Radar, Sonar \& Navigation, 11(6), pp. 892 - 902, (2017).

[25]. Lombardi, F., Griffiths, H. D., Balleri, A. "Influence of internal structure on landmine radar signatures", 13th European Radar Conference, pp. 161-164, (2016).

[26]. Lualdi, M., Lombardi, F. "Significance of GPR polarisation for improving target detection and characterisation". Nondestructive Testing and Evaluation, 29(4), pp. 345 - 356, (2014).

[27]. Lualdi, M., Zanzi, L. "Testing a safe acquisition procedure for an effective application of GPR to security operations". 18th Symposium on Application of Geophysics to Engineering and Environmental Problems, Atlanta, USA, pp. 613623, (2005).

[28]. Kang, W., Kim, C. R., Kim, J. H., Park, S. G., Cho, S. J., Son, J. S., Kim, K. W. "A study of antenna configuration for bistatic ground-penetrating radar", 16th International Conference on GPR, pp. 1-4, (2016)

[29]. Diamanti, N., Annan, A. P. "Characterizing the energy distribution around GPR antennas", Journal of Applied Geophysics, 99, pp. 83-90, (2013).

[30]. Eide, E., Vland, P.A., Sala, J. "Ground-coupled antenna array for step-frequency GPR", 15th International Conference on Ground Penetrating Radar, Brussels, pp. 756-761, (2014).

[31]. Lopera, O., Milisavljevi, N., Lambot, S. "Clutter reduction in GPR measurements for detecting shallow buried landmines: a Colombian case study", Near Surface Geophysics, 5(1), pp. 57-64, (2007).

[32]. Hines, M. J., Piers, A., Du, K., Gonzalez-Valdes, B., Martnez-Lorenzo, J., Rappaport, C. M., "Localization of anti-personnel landmines using multi-bistatic ground-coupled ground penetrating radar", IEEE Radio Science Meeting, pp. 241-241, (2014).

[33]. Zhang, Y., Orfeo, D., Burns, D., Miller, J., Huston, D., Xia, T. "Buried nonmetallic object detection using bistatic ground penetrating radar with variable antenna elevation angle and height". SPIE Smart Structures and Materials, Nondestructive Evaluation and Health Monitoring,pp.1016908-1016908, (2017)

[34]. Yilmaz, Ö. 'Seismic data analysis', Society of exploration geophysicists, (2001).

[35]. Collaborative ORDnance data repository, James Madison University, http://ordata.info/. 